Palynology

\title{
Pollen morphology of Rutaceae from Brazilian forest fragments
}

\section{Fernanda Vitorete Dutra \& Eduardo Custódio Gasparino}

To cite this article: Fernanda Vitorete Dutra \& Eduardo Custódio Gasparino (2018) Pollen morphology of Rutaceae from Brazilian forest fragments, Palynology, 42:1, 43-54, DOI: 10.1080/01916122.2017.1306812

To link to this article: https://doi.org/10.1080/01916122.2017.1306812

曲 Published online: 05 May 2017.

Submit your article to this journal

LII Article views: 151

View Crossmark data $\asymp$

Citing articles: 1 View citing articles 


\title{
Pollen morphology of Rutaceae from Brazilian forest fragments
}

\author{
Fernanda Vitorete Dutra and Eduardo Custódio Gasparino (iD) \\ Universidade Estadual Paulista, Unesp, Faculdade de Ciências Agrárias e Veterinárias, Departamento de Biologia aplicada à Agropecuária, Laboratório \\ de Morfologia Vegetal e Palinologia, Via de Acesso Prof. Paulo Donato Castellane s/n, Vila Industrial, Jaboticabal, SP, 14884900, Brazil
}

\section{ABSTRACT}

This paper presents pollen morphology of 12 native Brazilian species of Rutaceae from forest fragments of São Paulo, Brazil. The aim of this research is to expand the morphological knowledge of the species, thus contributing to taxonomic knowledge and, subsequently, conservation of species and the forest area. The pollen grains were acetolyzed, measured, described qualitatively, and illustrated using light microscopy (LM) and scanning electron microscopy (SEM). The studied species have differences in polarity, size, shape, aperture and exine ornamentation of the pollen grains, confirming the eurypalynous character of Rutaceae pollen. In particular, species of Zanthoxylum L. also presented significant differences in the pollen grains, confirming the morphological variation within the genus. The quantitative data and multivariate analysis confirm the morphological description; thus, the results reinforce the importance of pollen morphology in the identification and characterization of species of Rutaceae.

\section{KEYWORDS}

Eurypalynous; multivariate analysis; palynotaxonomy; pollen grains

\section{Introduction}

Currently, global biodiversity is being threatened due to fragmentation and loss of natural environments (Fahrig 2003). In Brazil the primary cause of this fragmentation is the expansion of agricultural frontiers, especially in the Cerrado, where the flora is characterized by high levels of endemism (Myers et al. 2000; Ribeiro \& Walter 2008).

Ranga et al. (2012) characterized the floristic structure of the native vegetation remnants from the northwest region of São Paulo, Brazil, searching for data about the occurrence of native species in these areas as well as the presence of endangered species in order to protect them and the conservation of biodiversity in these priority areas.

Rutaceae, with approximately 2040 species in 150-170 genera, is an important family of aromatic species with essential oils, native in the tropics and temperate regions of the Southern Hemisphere, especially South Africa and Australia (Kubitzki et al. 2011; Morton \& Telmer 2014). The family is positioned in Sapindales, along with other eight families (APG IV 2016). In Brazil, Rutaceae is represented by 194 species divided into 33 genera, of which 106 species are endemic. These species can be found in all Brazilian biomes. In the Cerrado the family has 41 species and 13 genera (Pirani \& Groppo 2016), and its specimens are usually associated with forest fragments, as indicated by Ranga et al. (2012).

The presence of a superior ovary, sieve tubes with S-type plastids, secretory cavities containing aromatic ethereal oils in the pericarp and parenchyma, and exstipulate leaves are morphological features that characterize Rutaceae (Chase et al. 1999). The placement of the family in the order Sapindales is strongly supported by molecular data (Gadek et al. 1996); however, despite several studies also carried out with molecular data the relationships between the genera of Rutaceae are not yet clear (Chase et al. 1999; Scott et al. 2000; Morton et al. 2003; Poon et al. 2007; Groppo et al. 2008; Bayer et al. 2009; Morton 2009; Razafimandimbison et al. 2010; Appelhans et al. 2011), and further studies are needed to confirm the monophyly of the family (Morton \& Telmer 2014).

The recent study of Rutaceae taxonomy by Morton \& Telmer (2014) presents a molecular phylogeny for the six previously proposed subfamilies of the family.The authors showed a new classification for Rutaceae, confirming the monophyly of four subfamilies: Aurantioideae, Cneoroideae, Rutoideae and Amyridoideae. The authors also point out that for the establishment of a natural and stable classification of the family, new information about other taxa is needed, combining molecular and morphological data (Morton \& Telmer 2014).

The pollen morphology of different species of Rutaceae has been described by Erdtman (1952), Sato (1971), Barth (1980, 1982, 1983, 1985), Arreguin-Sanchez et al. (1986), Roubick \& Moreno (1991), Corrêa et al. (1992), Mziray (1992), Breis et al. (1993), Morton \& Kallunki (1993), Victor \& Van Wyk (1998, 1999a, 1999b, 2000, 2001), Grant et al. (2000), Carreira \& Barth (2003), Qaiser \& Perveen (2005), Magalhães-e-Silva (2007), Fukuda et al. (2008), Mou \& Zhang (2009), Groppo et al. (2010), Cao et al. (2014), Radaeski et al. (2014), Silva et al. (2014) and Al-Anbari et al. (2015). These authors observed the differences in the morphology of the pollen grains of the species studied, especially regarding the aperture, shape and ornamentation of the exine, concluding that the family is eurypalynous.

Pollen studies on native species from remaining forest fragments indicate variations in shape, aperture, amb, ornamentation and size of the pollen grain (Souza \& Gasparino 2014; Belonsi \& Gasparino 2015), so the aim of this study is to identify 
morphological characteristics in pollen grains of the Rutaceae species which may be used for future taxonomic and palynological studies contributing to the conservation of these species in priority conservation areas.

\section{Material and methods}

We studied the pollen grains of 12 Rutaceae native species (see Appendix 1) from the remnant forest fragments of the northwest state of São Paulo described by Ranga et al. (2012). These species occur throughout the Brazilian territory, mainly concentrated in the Southeast, São Paulo state, in forest fragment areas (Figure 1, Specieslink 2017). We analyzed seven genera (Balfourodendron Mello ex Oliv., Esenbeckia Kunth., Galipea Aubl., Helietta Tul., Metrodorea A.St.-Hil., Pilocarpus Vahl and Zanthoxylum L.). Each genus is represented by one species, except
Zanthoxylum which has six species. Zanthoxylum rhoifolium Lam. is the most widely distributed in the sampled forest fragments. All Rutaceae species listed in Ranga et al. (2012) are new records in the study area. The pollen material was obtained from samples collected mainly in the remnant forest fragments of northwest São Paulo State, deposited as dried herbarium specimens in JABU and SJRP herbaria (acronym by Thiers 2017). When necessary, we used collections from other locations. Pollen grains of 20 different specimens were studied by light microscopy (LM) and scanning electron microscopy (SEM). The pollen grains of Zanthoxylum monogynum A.St.-Hil., Zanthoxylum petiolari A. St.-Hill. \& Tul. and Zanthoxylum rigidum Humb. \& Bonpl. ex Willd. had not been studied previously.

For LM analysis, permanents slides were prepared with pollen material acetolyzed according to Erdtman (1960) modified by Melhem et al. (2003). The pollen grains were measured (pollen

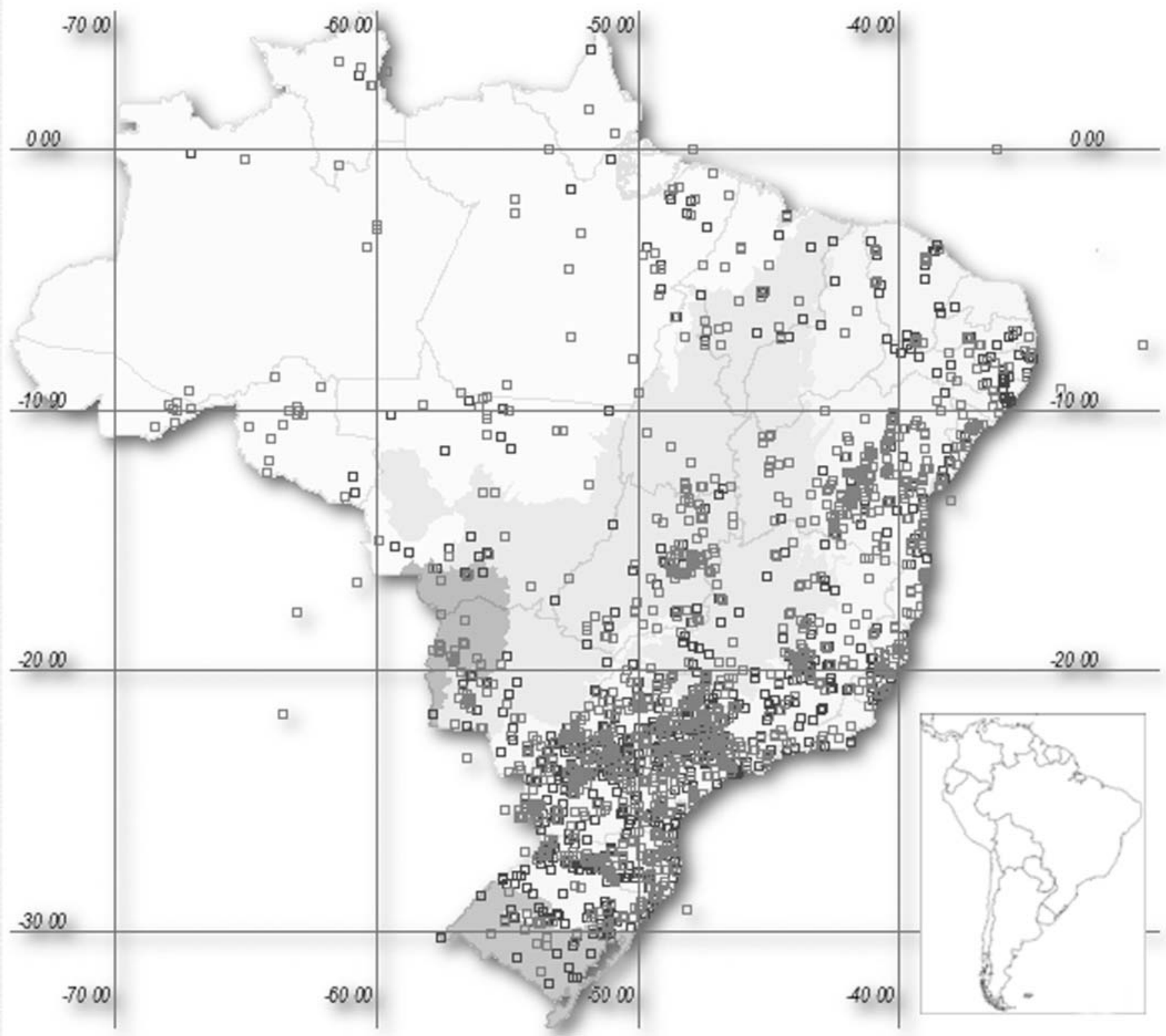

Figure 1. Occurrence map of the analyzed species of Rutaceae in Brazil. The squares correspond to the localities of collections available in the Specieslink database (http://splink.cria.org.br/). 
diameter, $\mathrm{n}=25$; aperture and exine thickness, $\mathrm{n}=10$ ) 7 days after preparation as established by Salgado-Labouriau et al. (1965). For SEM we used non-acetolyzed pollen grains, following Melhem et al. (2003). The slides were deposited in the pollen reference collection of the Plant Morphology and Palynology Laboratory, Departamento de Biologia aplicada à Agropecuária, Universidade Estadual Paulista, Unesp, Jaboticabal, Brazil.

A statistical analysis was conducted with the measures taken to obtain the means $(\mathrm{x})$, standard deviation (sx), standard error (s), 95\% confidence intervals (Cl), coefficient of variability (V) and range (R) according to Vieira (2011) and Zar (1999). For measures of auxin thickness, and length and width of apertures, only the arithmetic mean was calculated. To compare the values of the pollen grain diameters we used the graphs of the MINITAB program which represent the mean and the confidence interval values. A principal component analysis (PCA) was performed using the programs FITOPAC (Shepherd 1996) and PC-ORD (McCune \& Mefford 2011). We used eight metric variables for the PCA: length of colpus (CLEN), width of colpus (CWID), polar diameter in equatorial view (PDIA), equatorial diameter in equatorial view (EDIA), length of endoaperture (ELEN), width of endoaperture (EWID), shape (SHAP) and exine (EXIN).

The pollen description and terminology follows Barth \& Melhem (1988) and Punt et al. (2007); we adopted the polar area index proposed by Faegri \& Iversen (1966), and the colpi width index is according to Gasparino et al. (2013). Photomicrographs were taken with a Bel Photonics light microscope, for LM photos, and with a JEOL. JSM5410 scanning electron microscope for the SEM images.

\section{Results}

\subsection{General description}

The pollen grains of the Rutaceae species studied here (Plates 1-3) are monads, apolar or isopolar, small to large (Tables 1-3), with circular to subcircular amb, oblate-spheroidal to prolate, inaperturate, 3-colporate or 4-colporate, with long colpi, narrow or wide, with or without margo, rounded or tapered at the polar ends, endoaperture lalongate, microreticulate, reticulate, striate-microreticulate or striate-reticulate exine, sometimes with differences between apocolpium and mesocolpium, sexine thicker than nexine.

\subsection{Species description}

Balfourodendron riedelianum (Engl.) Engl. (Plate 1 figures 1-5; Tables 1, 3, 4)

Isopolar pollen grains, small to medium $(27.66 \times 21.49 \mu \mathrm{m})$, circular amb, subprolate, 3-colporate, long and narrow colpi rounded at the ends, with margo, lalongate endoapertures, microreticulate homobrochate exine (Plate 1, figure 3).

Esenbeckia febrifuga (A. St.-Hill.) A. Juss ex Mart (Plate 1, figures 6-10; Tables 1, 3, 4)

Isopolar pollen grains, medium $(29.38 \times 25.21 \mu \mathrm{m})$, circular amb, subprolate, 3-colporate, long and narrow colpi, without margo, lalongate endoapertures tapered at the ends, striate- microreticulate exine (Plate 1, figure 10) with microreticulum homobrochate in mesocolpium (Plate 1, figure 6).

Galipea jasminiflora (A. St.-Hill.) Engl. (Plate 1, figures 11-13; Tables 1, 2, 4)

Apolar pollen grains, large $(72.60 \times 67.80 \mu \mathrm{m})$, circular amb, prolate-spheroidal, inaperturate, reticulate heterobrochate exine, very large lumina, sinuous muri and bacula inside lumina (Plate 1, figures 12-13).

Helietta apiculata Benth. (Plate 1, figures 14-20; Tables 1, 3, 4) Isopolar pollen grains, medium $(29.80 \times 25.60 \mu \mathrm{m})$, circular amb, subprolate, 3-colporate, long and narrow colpi, with margo, lalongate endoapertures and tapered at the ends, striate-microreticulate exine (Plate 1, figures 19-20) with microreticulum homobrochate in mesocolpium (Plate 1, figure 14).

Metrodorea nigra A. St.-Hill. (Plate 2, figures 1-4; Tables 1, 3, 4)

Isopolar pollen grains, medium $(37.10 \times 29.10 \mu \mathrm{m})$, subcircular amb, subprolate, 3-colporate, long and narrow colpi, with margo, lalongate endoapertures tapered at the ends, striatemicroreticulate exine in apocolpium and mesocolpium (Plate 2, figures 1, 4).

Pilocarpus spicatus A. St.-Hill. (Plate 2, figures 5-9; Tables 1, 3, 4)

Isopolar pollen grains, small to medium $(30.00 \times 16.50 \mu \mathrm{m})$, circular amb, prolate, 3-colporate, long and narrow colpi, without margo, lalongate endoapertures (Plate 2, figures 5-7), striate-reticulate exine with reticula heterobrochate in mesocolpium (Plate 2, figures 8-9) and apocolpium striatereticulate.

Zanthoxylum caribaeum Lam. (Plate 2, figures 10-14; Tables 1, 3, 4)

Isopolar pollen grains, small to medium $(26.43 \times 18.51 \mu \mathrm{m})$, subcircular amb, prolate, 3-colporate, long and wide colpi (Plate 2 , figures 11-12), with margo, lalongate endoapertures tapered at the ends, striate-reticulate exine in apocolpium and mesocolpium (Plate 2, figures 13-14).

Zanthoxylum monogynum A.St.-Hil. (Plate 2, figures 15-19; Tables 1, 3, 4)

Isopolar pollen grains, small $(17.38 \times 15.53 \mu \mathrm{m})$, subcircular amb, prolate-spheroidal, 3-colporate, long and wide colpi, without margo, lalongate endoapertures (not evident), striate-reticulate exine with reticula homobrochate in mesocolpium (Plate 2 , figures 16,18 ) and apocolpium striate-reticulate.

Zanthoxylum petiolari A. St.-Hill. \& Tul. (Plate 2, figure 20, Plate 3, figures 1-4; Tables 1, 3, 4)

Isopolar pollen grains, small $(20.57 \times 18.92 \mu \mathrm{m})$, circular amb, oblate-spheroidal, 3-colporate, long and narrow colpi, without margo, lalongate endoapertures tapered at the ends, striate-reticulate exine with reticula homobrochate in mesocolpium (Plate 3, figure 3) and apocolpium striate-reticulate (Plate 2, figure 20).

Zanthoxylum rhoifolium Lam. (Plate 3, figures 5-9; Tables 1, 3, 4) Isopolar pollen grains, small to medium $(23.10 \times 24.60 \mu \mathrm{m})$, circular amb, oblate-spheroidal, 4-colporate (Plate 3, figure 5), long and narrow colpi, without margo, lalongate endoapertures tapered at the ends, striate-reticulate exine with reticula heterobrochate in apocolpium (Plate 3, figure 5) and mesocolpium striate-reticulate (Plate 3, figures 8-9). 

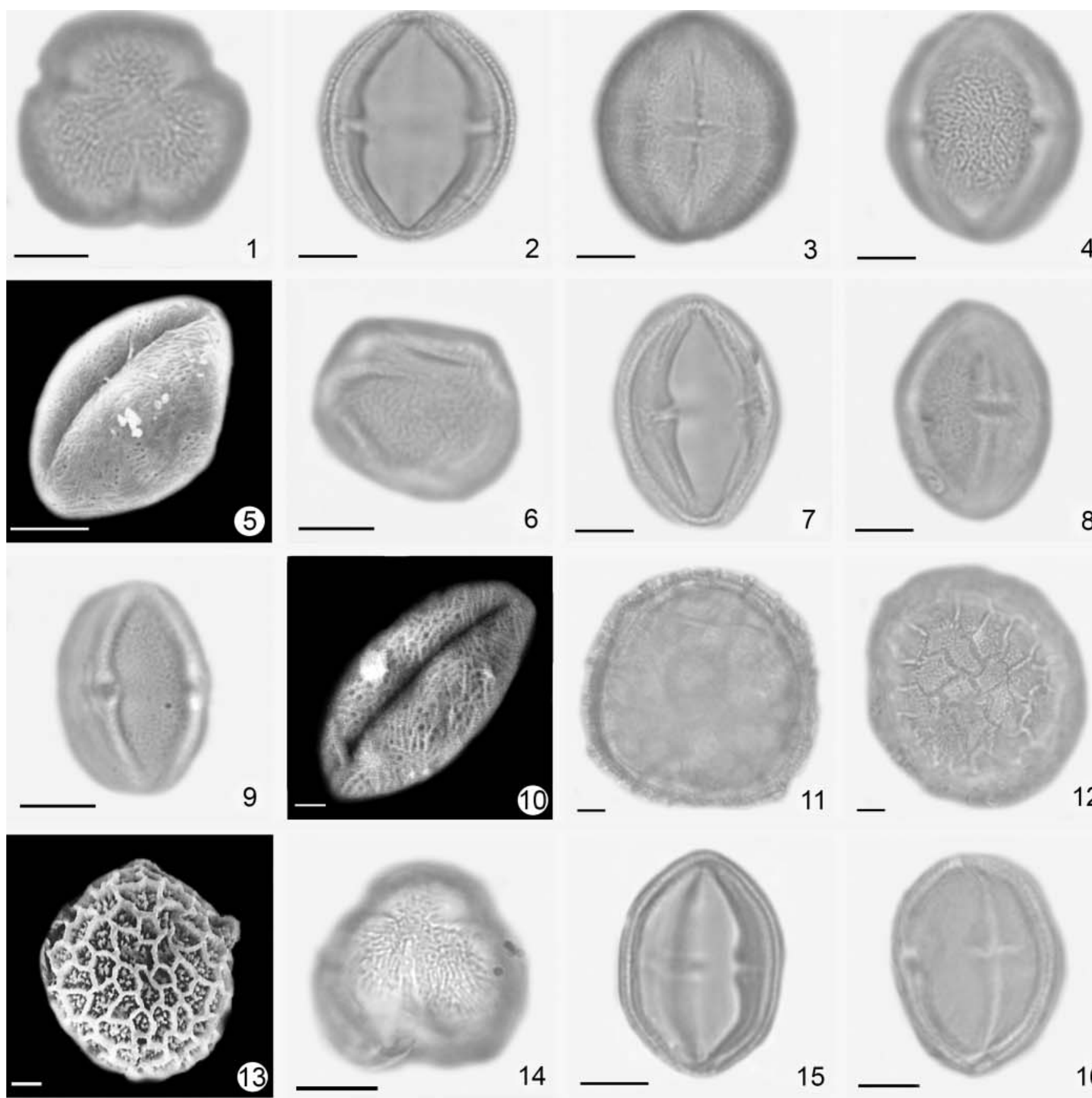

15
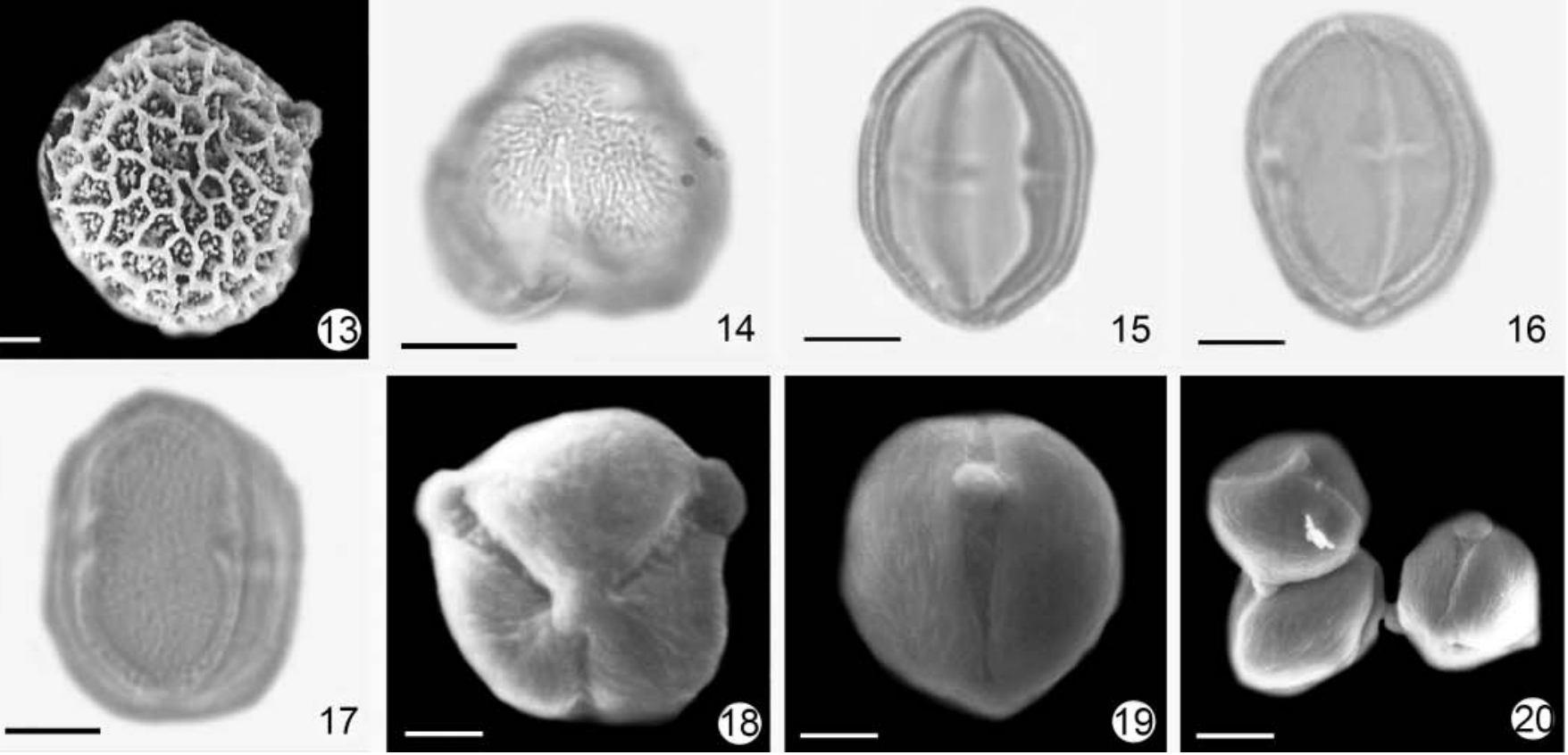

Plate 1. Photomicrographs and scanning electron micrographs (SEM) of the pollen grains of Rutaceae A. Juss. Figures 1-5. Balfourodendron riedelianum (Engl.) Engl. 1. General aspect, polar view. 2. Polar view, opitical section. 3. Equatorial view, aperture. 4. Equatorial view, ornamentation. 5. Equatorial view, in SEM. Figures 6-10. Esenbeckia febrifuga (A. St.-Hill.) A. Juss ex Mart. 6. General aspect, polar view. 7. Equatorial view, optical section. 8. Equatorial view, aperture. 9. Equatorial view, ornamentation. 10. Equatorial view, in SEM. Figures 11-13. Galipea jasminiflora (A. St.-Hill.) Engl. 11. General aspect, optical section. 12. General aspect, ornamentation. 13. General aspect, ornamentation in SEM. Figures 14-20. Helietta apiculata Benth. 14. General aspect, polar view. 15. Equatorial view, optical section. 16. Equatorial view, aperture. 17. Equatorial view, ornamentation. 18. Polar view, in SEM. 19. Equatorial view, in SEM. 20. General aspect, in SEM. Scale bars: $1-9,11-17=10 \mu \mathrm{m} ; 10,18-20=5 \mu \mathrm{m}$. 

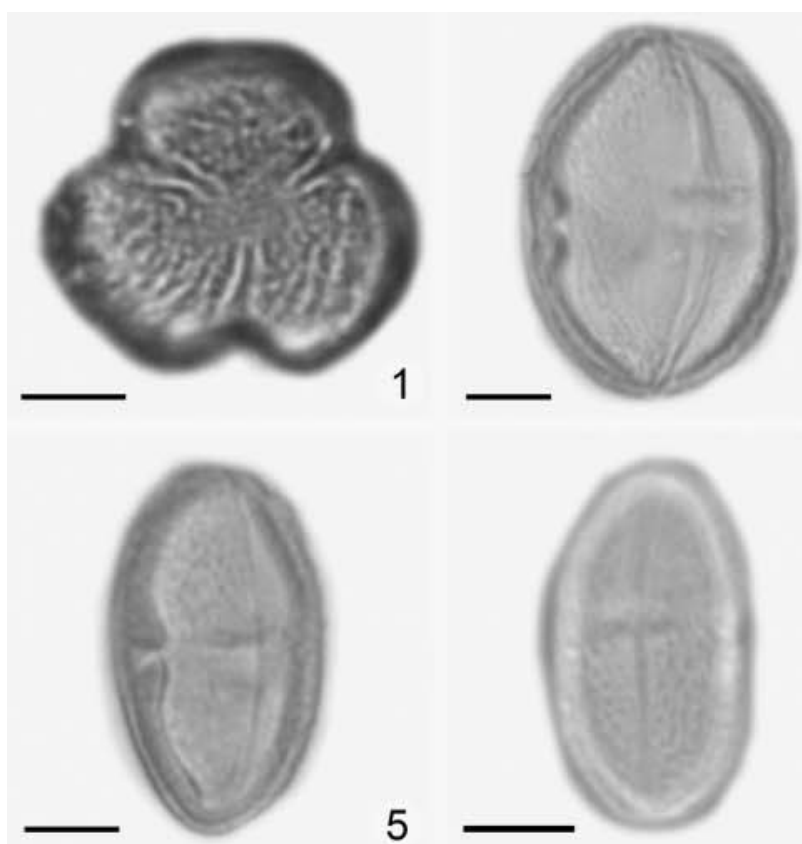

5

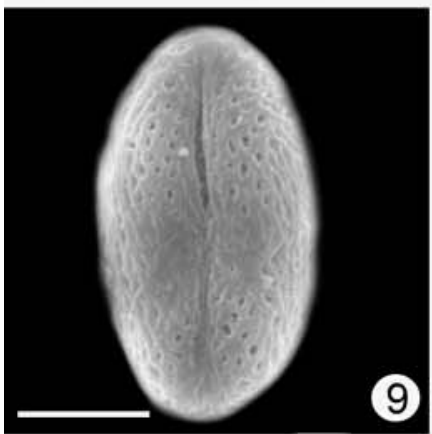

(9)

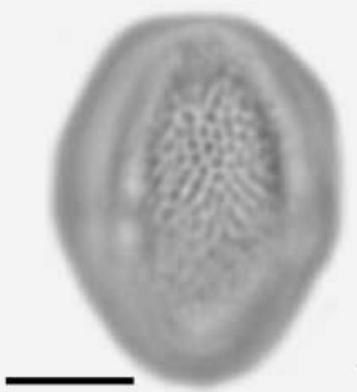

13
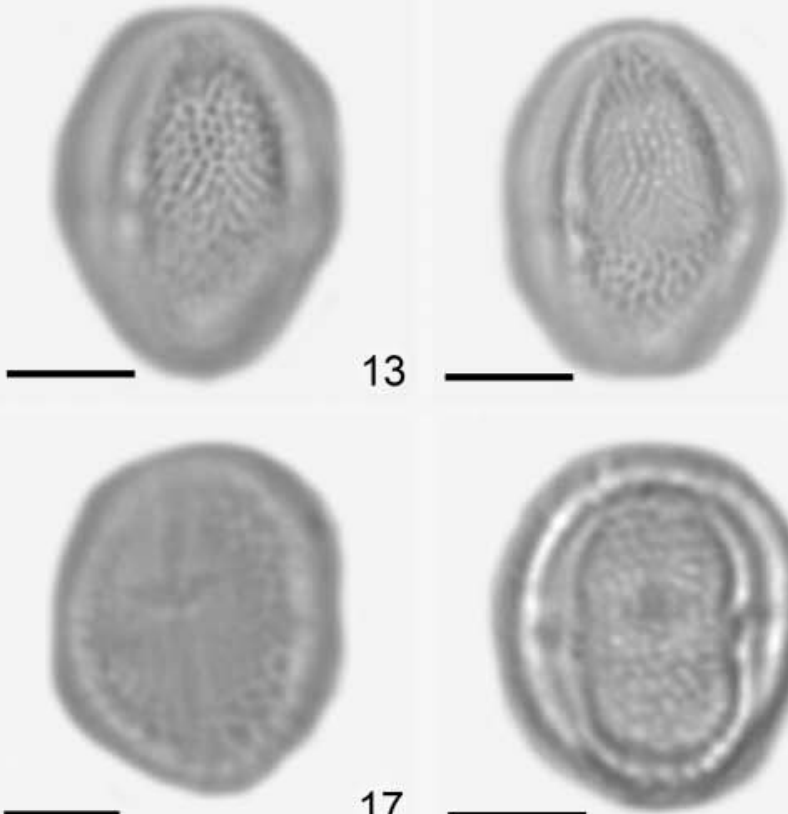

17
2

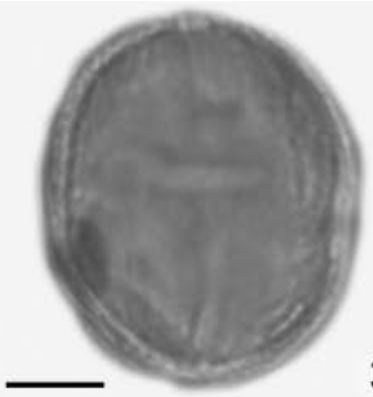

3

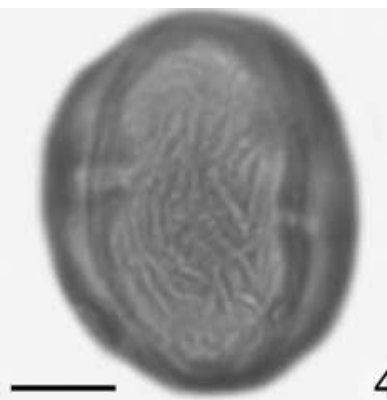

6

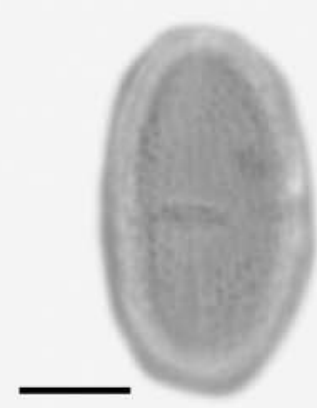

7

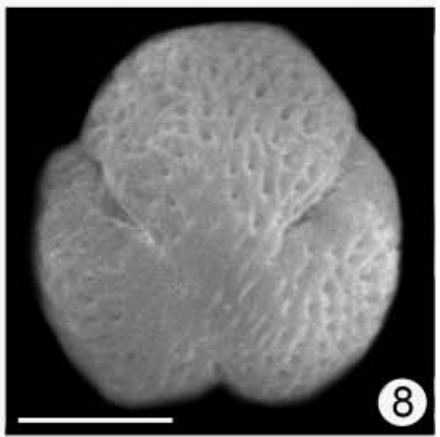

11

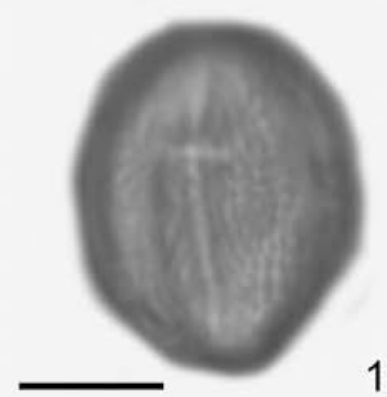

12
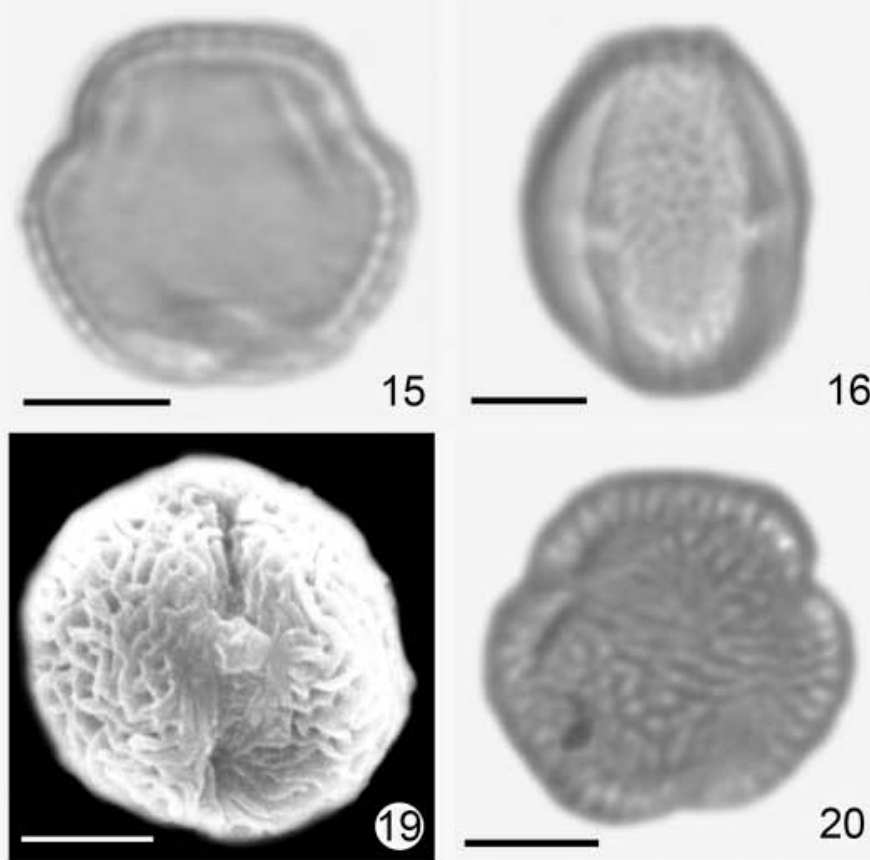

Plate 2. Photomicrographs and scanning electron micrographs (SEM) of the pollen grains of Rutaceae A. Juss. Figures 1-4. Metrodorea nigra A. St.-Hill. 1. General aspect, polar view. 2. Equatorial view, optical section. 3. Equatorial view, aperture. 4. Equatorial view, ornamentation. Figures 5-9. Pilocarpus spicatus A. St.-Hill. 5. General aspect, equatorial view. 6. Equatorial view, aperture. 7. Equatorial, view, ornamentation. 8. Polar view, in SEM. 9. Equatorial view, in SEM. Figures 10-14. Zanthoxylum caribaeum (A. St.-Hill. \& Tul.) Reynel. 10. General aspect, polar view. 11. General aspect, equatorial view. 12. Equatorial view, aperture. 13. Equatorial view, ornamentation in high focus. 14. Equatorial view, ornamentation in low focus. Figures 15-19. Zanthoxylum monogynum A. St.-Hill. 15. General aspect, polar view. 16. Equatorial view, optical section. 17. Equatorial view, aperture. 18. Equatorial view, ornamentation. 19. Equatorial view, in SEM. Figure 20. Zanthoxylum petiolari A. St.-Hill. \& Tul. Polar view, ornamentation. Scale bars $=10 \mu \mathrm{m}$. 

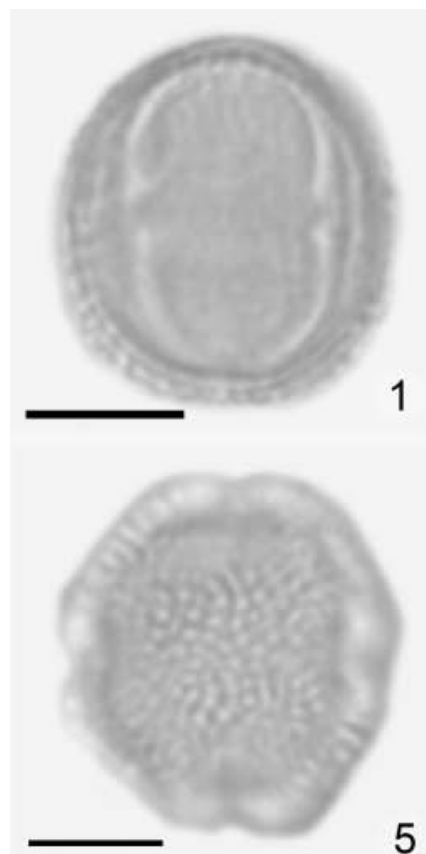

5
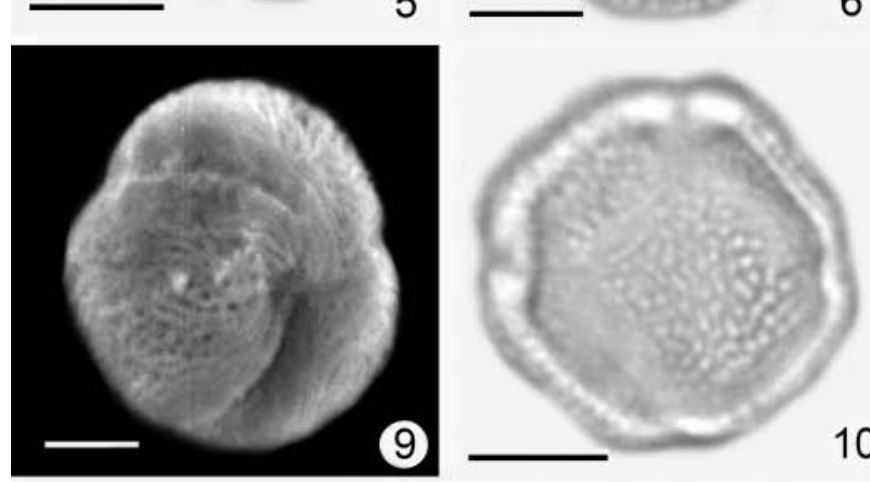

13
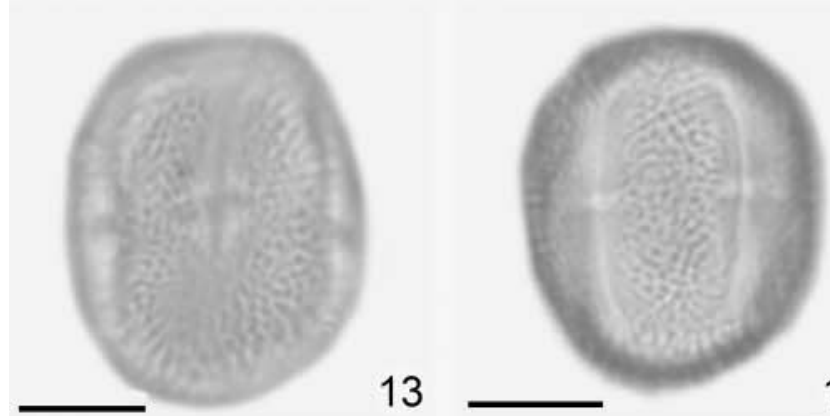

14

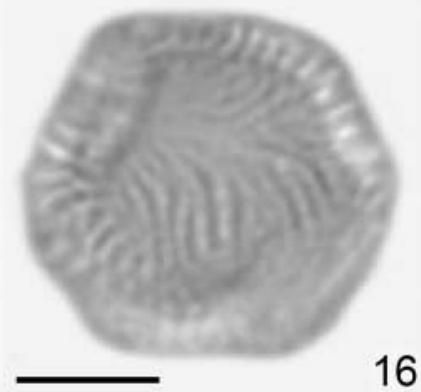

3

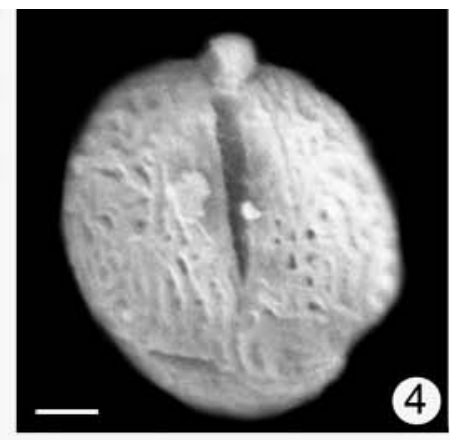

7

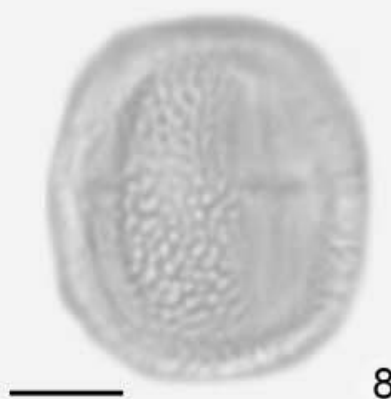

8
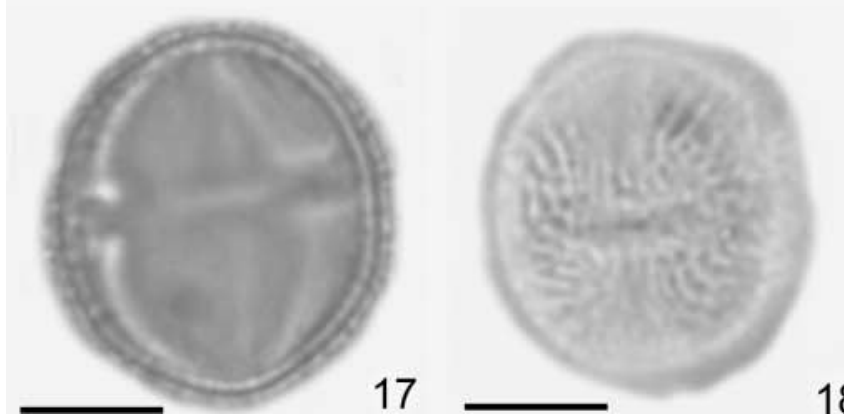

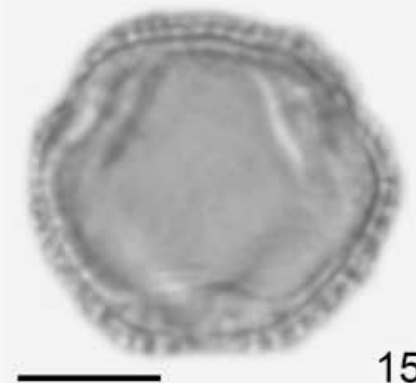

6

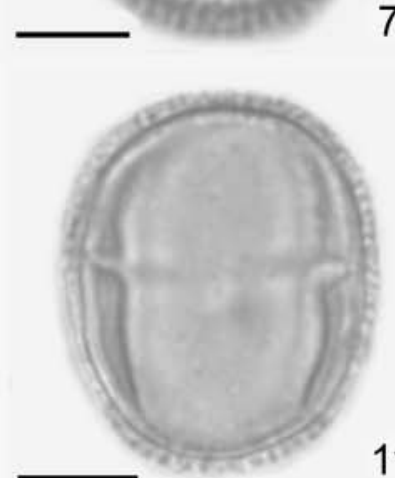

11

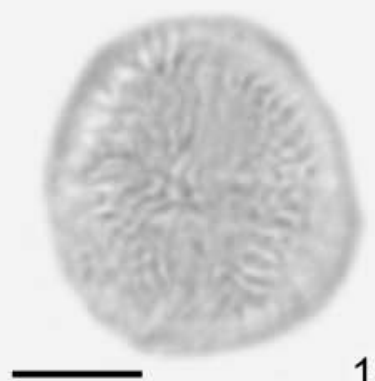

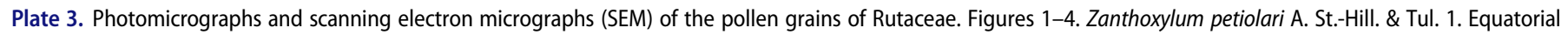

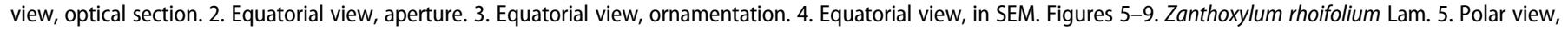

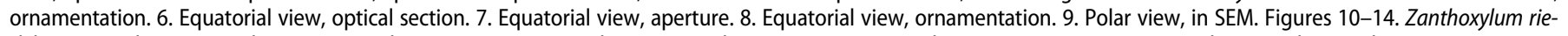
delianum Engl. 10. General aspect, optical section. 11. Equatorial view, optical section. 12. Equatorial view, aperture. 13 . Equatorial view, colpus and ornamentation. 14.

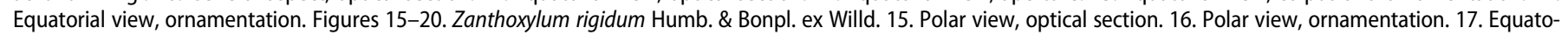

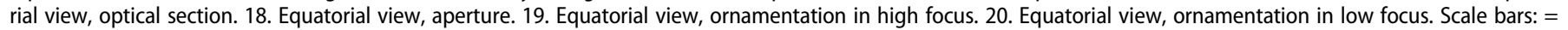
$1-3,5-8,10-20=10 \mu \mathrm{m} ; 4,9=5 \mu \mathrm{m}$. 
Table 1. Morphological characterization of Rutaceae pollen grains ( $P / E=$ ratio between polar and equatorial diameter; $\mathrm{AN}=$ aperture number; $\mathrm{EO}=$ exine ornamentation; $S=$ small; $M=$ medium; $L=$ large; $O S=$ oblate spheroidal; $P R=$ prolate; $\mathrm{PS}=$ prolate spheroidal; $\mathrm{SP}=$ subprolate; $\mathrm{MR}=$ microreticulate; $\mathrm{SM}=$ striatemicroreticulate; $\mathrm{SR}=$ striate-reticulate; $\mathrm{RT}=$ reticulate; $\mathrm{ST}=$ striate).

\begin{tabular}{lccccc}
\hline Species & Size & P/E & Shape & AN & EO \\
\hline Balfourodendron riedelianum & S-M & 1.29 & SP & 3 & MR \\
Esenbeckia febrifuga & $\mathrm{M}$ & 1.17 & SP & 3 & SM \\
Galipea jasminiflora & $\mathrm{L}$ & 1.07 & $\mathrm{PS}$ & 0 & $\mathrm{RT}$ \\
Helietta apiculata & $\mathrm{M}$ & 1.16 & $\mathrm{SP}$ & 3 & $\mathrm{SM}$ \\
Metrodorea nigra & $\mathrm{M}$ & 1.27 & $\mathrm{SP}$ & 3 & $\mathrm{SM}$ \\
Pilocarpus spicatus & $\mathrm{S}-\mathrm{M}$ & 1.82 & $\mathrm{PR}$ & 3 & $\mathrm{SR}$ \\
Zanthoxylum caribaeum & $\mathrm{M}$ & 1.43 & $\mathrm{PR}$ & 3 & $\mathrm{SR}$ \\
Zanthoxylum monogynum & $\mathrm{S}$ & 1.12 & $\mathrm{PS}$ & 3 & $\mathrm{SR}$ \\
Zanthoxylum petiolari & $\mathrm{S}$ & 0.92 & $\mathrm{OS}$ & 3 & $\mathrm{SR}$ \\
Zanthoxylum rhoifolium & $\mathrm{M}$ & 0.94 & $\mathrm{OS}$ & 4 & $\mathrm{SR}$ \\
Zanthoxylum riedelianum & $\mathrm{M}$ & 1.18 & $\mathrm{SP}$ & 4 & $\mathrm{SM}$ \\
Zanthoxylum rigidum & $\mathrm{S}-\mathrm{M}$ & 1.09 & $\mathrm{PS}$ & 3 & $\mathrm{SR}$ \\
\hline
\end{tabular}

Table 2. Quantitative data of inaperturate pollen grains of Rutaceae, $n=25$.

\begin{tabular}{lcccc}
\hline Species & $(\mathrm{R}) x \pm s_{x}$ & $s$ & $\mathrm{Cl}$ & $\mathrm{V}$ \\
\hline $\begin{array}{l}\text { Galipea jasminiflora } \\
\text { Diameter I }\end{array}$ & $(62.50-87.50) 72.60 \pm 0.97$ & 4.87 & $(70.59-74.61)$ & 6.70 \\
Diameter II & $(62.50-75.00) 67.80 \pm 0.86$ & 4.29 & $(66.03-69.57)$ & 6.33 \\
\hline
\end{tabular}

$R$ range, $x$ mean $(\mu \mathrm{m}), s_{x}$ standard deviation $(\mu \mathrm{m}), s$ standard error $(\mu \mathrm{m})$,

$\mathrm{Cl}$ confidence interval in $95 \%(\mu \mathrm{m}), V$ coefficient of variability (\%).

Zanthoxylum riedelianum Engl. (Plate 3, figures 10-14; Tables 1, $3,4)$

Isopolar pollen grains, small to medium $(27.15 \times 22.93 \mu \mathrm{m})$, circular amb, subprolate, 4-colporate (Plate 3, figure 10), long and narrow colpi, without margo, lalongate endoapertures tapered at the ends, striate-microreticulate exine with

Table 3. Quantitative data of aperturate pollen grains of Rutaceae, $n=25$.

\begin{tabular}{|c|c|c|c|c|}
\hline Species & (R) $x \pm s_{x}$ & $S$ & $\mathrm{Cl}$ & V \\
\hline \multicolumn{5}{|c|}{ Polar diameter in equatorial view } \\
\hline B. riedelianum & (23.14-30.85) $27.66 \pm 0.43$ & 2.14 & $(26.78-28.54)$ & 07.72 \\
\hline E. febrifuga & (25.00-32.50 $29.38 \pm 0.01$ & 2.17 & $(29.36-29.39)$ & 07.37 \\
\hline H. apiculata & (25.00-37.50 $29.80 \pm 0.72$ & 3.60 & $(28.32-31.28)$ & 12.09 \\
\hline M. nigra & $(32.50-42.50) 37.10 \pm 0.57$ & 2.86 & $(35.92-38.28)$ & 07.70 \\
\hline P. spicatus & $(25.00-37.50) 30.00 \pm 0.78$ & 3.89 & $(28.40-31.60)$ & 12.95 \\
\hline Z. caribaeum & $(23.14-33.42) 26.43 \pm 0.58$ & 2.92 & $(25.23-27.63)$ & 11.06 \\
\hline Z. monogynum & (15.43-20.57) $17.38 \pm 0.37$ & 1.86 & $(16.61-18.15)$ & 10.70 \\
\hline Z. petiolari & (15.43-23.14) $20.57 \pm 0.42$ & 2.10 & $(19.70-21.43)$ & 10.21 \\
\hline Z. rhoifolium & (18.75-27.50) $23.10 \pm 0.47$ & 2.34 & $(22.14-24.06)$ & 10.14 \\
\hline Z. riedelianum & (23.14-30.85) $27.15 \pm 0.45$ & 2.24 & $(26.23-28.07)$ & 08.24 \\
\hline Z. rigidum & (22.50-30.00) $26.00 \pm 0.32$ & 1.61 & $(25.34-26.66)$ & 06.21 \\
\hline \multicolumn{5}{|c|}{ Equatorial diameter in equatorial view } \\
\hline B. riedelianum & (18.00-25.71) $21.49 \pm 0.43$ & 1.95 & $(20.69-22.30)$ & 09.06 \\
\hline E. febrífuga & $(22.50-30.00) 25.21 \pm 0.57$ & 1.98 & $(24.03-26.39)$ & 07.86 \\
\hline H. apiculata & (20.00-30.00 $25.60 \pm 0.42$ & 2.08 & $(24.74-26.46)$ & 08.11 \\
\hline M. nigra & $(25.00-35.00) 29.10 \pm 0.58$ & 2.88 & $(27.92-30.28)$ & 09.88 \\
\hline P. spicatus & (12.50-20.00 $16.50 \pm 0.46$ & 2.28 & $(15.56-17.44)$ & 13.83 \\
\hline Z. caribaeum & (12.86-25.71) $18.51 \pm 0.65$ & 3.24 & $(17.18-19.84)$ & 17.48 \\
\hline Z. monogynum & (12.86-18.00 $15.53 \pm 0.35$ & 1.74 & $(14.81-16.24)$ & 11.19 \\
\hline Z. petiolari & (15.43-23.14) $18.92 \pm 0.39$ & 1.95 & $(18.12-19.72)$ & 10.29 \\
\hline Z. rhoifolium & $(22.50-27.50) 24.60 \pm 0.31$ & 1.56 & $(23.96-25.24)$ & 06.35 \\
\hline Z. riedelianum & (20.57-25.71) $22.93 \pm 0.39$ & 1.95 & $(22.13-23.74)$ & 08.51 \\
\hline Z. rigidum & (20.00-25.00 $23.80 \pm 0.36$ & 1.79 & $(23.06-24.54)$ & 07.50 \\
\hline
\end{tabular}

$R$ range, $x$ mean $(\mu \mathrm{m}), s_{x}$ standard deviation $(\mu \mathrm{m}), s$ standard error $(\mu \mathrm{m})$, $\mathrm{Cl}$ confidence interval in $95 \%(\mu \mathrm{m}), V$ coefficient of variability $(\%)$.
Table 4. Measurements (in $\mu \mathrm{m}$ ) of Rutaceae pollen grains, apertures and exine $(n=10)$

\begin{tabular}{lcccccccc}
\hline & \multicolumn{3}{c}{ Colpus } & & \multicolumn{2}{c}{ Endoaperture } & & \\
\cline { 2 - 3 } Species & Length & Width & & Length & Width & & Ex & WCI \\
\hline Balfourodendron riedelianum & 19.28 & 2.31 & & 1.62 & 9.00 & 1.30 & 9.30 \\
Esenbeckia febrifuga & 24.42 & 2.70 & & 2.57 & 12.60 & 2.57 & 9.33 \\
Galipea jasminiflora & - & - & - & - & 4.37 & - \\
Helietta apiculata & 20.17 & 2.57 & & 2.57 & 13.63 & 2.57 & 9.96 \\
Metrodorea nigra & 25.10 & 2.60 & & 2.70 & 13.70 & 2.70 & 11.19 \\
Pilocarpus spicatus & 25.30 & 1.00 & & 1.90 & 7.70 & 1.30 & 16.50 \\
Zanthoxylum caribaeum & 17.74 & 2.83 & & 2.57 & 7.46 & 2.57 & 6.54 \\
Zanthoxylum monogynum & 25.70 & 2.83 & & 2.55 & 16.71 & 3.00 & 5.48 \\
Zanthoxylum petiolari & 14.14 & 2.57 & & 2.57 & 9.38 & 2.70 & 7.36 \\
Zanthoxylum rhoifolium & 20.56 & 2.57 & & 2.44 & 10.80 & 2.57 & 9.57 \\
Zanthoxylum riedelianum & 16.71 & 1.89 & & 2.16 & 10.28 & 1.77 & 11.97 \\
Zanthoxylum rigidum & 14.39 & 2.57 & & 2.57 & 5.91 & 2.57 & 9.26 \\
\hline
\end{tabular}

Ex exine thickness, $W C I$ width colpus index.

microreticulum homobrochate in mesocolpium (Plate 3, figure 14) and apocolpium striate-microreticulate (Plate 3 , figure 10).

Zanthoxylum rigidum Humb. \& Bonpl. ex Willd. (Plate 3, figures 15-20; Tables 1, 3, 4)

Isopolar pollen grains, small to medium $(26.00 \times 23.80 \mu \mathrm{m})$, subcircular amb, prolate-spheroidal, 3-colporate, long and narrow colpi, without margo, lalongate endoapertures tapered at the ends, striate-reticulate exine in apocolpium and mesocolpium (Plate 3, figures 16, 20).

\subsection{Pollen key to Rutaceae species}

1. Apolar and inaperturate pollen grains Galipea jasminiflora

1 '. Isopolar and aperturate pollen grains ..................... 2

2. Pollen grains 4 -colporate ................................. 3

3. Oblate-spheroidal pollen grains with reticulate heterobrocate apocolpium

Zanthoxylum rhoifolium

3'. Subprolate pollen grains with striate-microreticulate apocolpium

Zanthoxylum riedelianum

2 '. Pollen grains 3 -colporate ................................ 4 4. Exine microreticulate ... Balfourodendron riedelianum 4'. Exine striate-reticulate or striate-microreticulate ...... 5

5. Ornamentation striate-microreticulate ...............6 6. Apocolpium and mesocolpium striate-microreticulate .................................. Metrodorea nigra

6'. Mesocolpium microreticulate ................... 7

7. Colpi without margo .........Esenbeckia febrifuga

7'. Colpi with margo ............... Helietta apiculata

$5^{\prime}$. Ornamentation striate-reticulate ................... 8

8. Apocolpium and mesocolpium striate-reticulate ... 9 9. Prolate pollen grains, wide colpi with margo Zanthoxylum caribaeum

9'. Prolate-spheroidal pollen grains, narrow colpi without margo ........... Zanthoxylum rigidum

$8^{\prime}$. Apocolpium and mesocolpium reticulate ...... 10

10. Heterobrochate reticula in mesocolpium Pilocarpus spicatus 

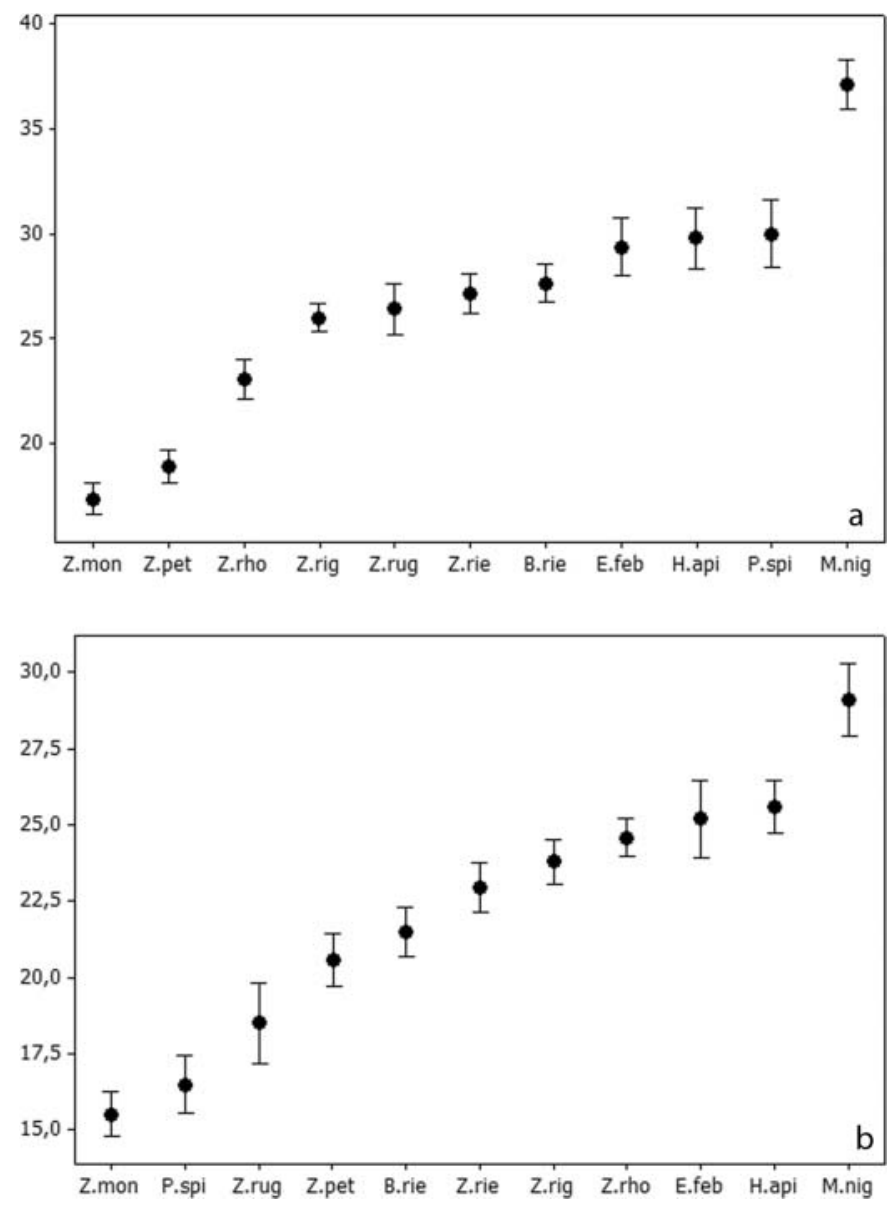

Figure 2. Representation of confidence interval of mean in $95 \%$ of the pollen grains of Rutaceae. (a) Polar diameter in equatorial view. (b) Equatorial diameter in equatorial view. The higher and lower boundaries show the confidence interval; the average circle shows the arithmetic mean. B.rie = Balfourodendron riedelianum; E.feb = Esenbeckia febrifuga; H.api = Helietta apiculata; M.nig = Metrodorea nigra P.spi = Pilocarpus spicatus; Z.car = Zanthoxylum caribaeum; Z.mon = Zanthoxylum monogynum; Z.pet = Zanthoxylum petiolari; Z.rho = Zanthoxylum rhoifolium; Z.rie $=$ Zanthoxylum riedelianum; Z.rig = Zanthoxylum rigidum. The values are in $\mu \mathrm{m}$.

10'. Homobrochate reticula in mesocolpium ............................................. 11

11. Prolate-spheroidal pollen grains, wide colpi Zanthoxylum monogynum

11'. Oblate-spheroidal pollen grains, narrow colpi Zanthoxylum petiolari

\subsection{Analysis of quantitative data}

For quantitative analysis of the pollen grain size we used the polar and equatorial diameters in equatorial view for the aperturate species (Figure 2a, b). Galipea jasminifora, with inaperturate pollen grains, was excluded from this comparison. When we observe the mean and confidence interval of the pollen grain diameters, we find that Zanthoxylum monogynum and Zanthoxylum petiolari are separated from the other species by lower polar diameters. The same applies to the Metrodorea nigra pollen grains; however, this species has higher values for the polar diameter. The other species are in a continuous group of intermediate diameter values.

When we consider the equatorial diameter, Metrodorea nigra has a larger diameter than all the other species. We can also observe a continuous group with all other species in which the Zanthoxylum species have the lowest values for this diameter (Figure 2a, b).

The PCA is an exploratory analysis of quantitative data valued for Rutaceae pollen grains. This analysis summarizes in its two axes (Figure 3) $94.42 \%$ of total data variability. The first axis of the PCA explained $89.88 \%$ of the total variability according to the metric variables. On this axis we note a separation in the ordination of the species, so that Galipea jasminiflora, the only species with large and inaperturate pollen grains, appears alone on the positive side of the first axis, in contrast to the others that are very close together on the negative side of this axis (except Zanthoxylum rigidum). The most relevant variables for the species grouping in the first axis were the length of the colpus (CLEN) and width of the endoaperture (EWID) (Table 5).

Although the contributions of the second PCA axis were much smaller than those of the first axis (only 4.54\%), we can

Table 5. Pearson and Kendall correlation coefficients for pollen grain metric variables of the first and the second axis of principal component analysis (PCA) ordination in Rutaceae species.

\begin{tabular}{lcr}
\hline & \multicolumn{2}{c}{ Principal components } \\
\cline { 2 - 3 } Variables & Axis 1 & Axis 2 \\
\hline EDIA & 0.2381 & -0.2882 \\
PDIA & 0.2171 & 0.1993 \\
CLEN & -0.6682 & 0.3400 \\
CWID & -0.2640 & -0.4772 \\
ELEN & -0.2661 & -0.2141 \\
EWID & -0.5501 & -0.2356 \\
EXIN & 0.0941 & -0.5893 \\
SHAP & -0.0122 & 0.2921 \\
\hline
\end{tabular}

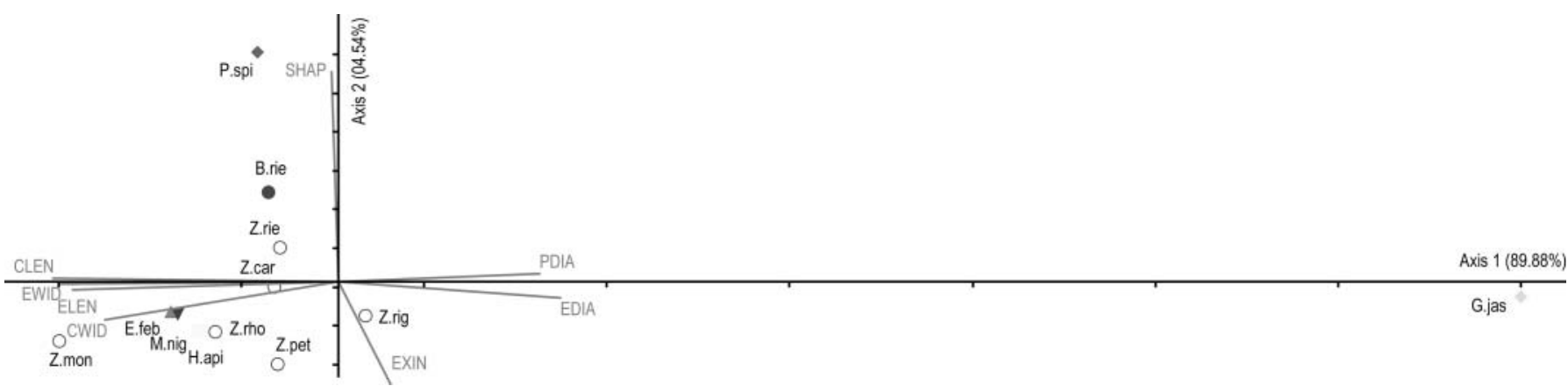

Figure 3. Principal component analysis performed with the pollen metrical variables from Rutaceae species. B.rie $=$ Balfourodendron riedelianum; E.feb $=$ Esenbeckia febrifuga; G.jas = Galipea jasminiflora; H.api = Helietta apiculata; M.nig = Metrodorea nigra; P.spi = Pilocarpus spicatus; Z.car = Zanthoxylum caribaeum; Z.mon = Zanthoxylum monogynum; Z.pet = Zanthoxylum petiolari; Z.rho = Zanthoxylum rhoifolium; Z.rie = Zanthoxylum riedelianum; Z.rig = Zanthoxylum rigidum. 
check the distance of Pilocarpus spicatus (on the positive side) from the other analyzed species. The values of exine (EXIN) and width of colpus (CWID) were the most important variables for the placement of the species along the second axis (Table 5).

The PCA of the Rutaceae species here analyzed confirms the qualitative data described for the pollen grains since only Galipea jasminiflora (with large and inaperturate pollen grains) is well separated from all other species. The high values in shape also contributed to the distance of Pilocarpus spicatus pollen grains from other species. Although important, the measurements obtained of pollen grains without the qualitative data contribute little to separate the genera and species.

\section{Discussion}

The pollen grains of the Rutaceae generally present variations in shape, aperture number and type, and exine ornamentation, as portrayed in previous studies (Morton \& Kallunki 1993; Grant et al. 2000; Fukuda et al. 2008). They are usually colporate (rarely porate as in Spiranthera A.St.-Hil. or pantoaperturate as in Almeidea A.St.-Hil.), with three to seven apertures and microreticulate, reticulate, striate, striate-reticulate or, rarely, psilate exine, such as in Hortia Vand. species (Morton \& Kallunki 1993; Groppo et al. 2010). The Rutaceae genera here analyzed were previously reported by Barth (1980, 1982, 1985), Corrêa et. al. (1992), Roubik \& Moreno (1991), Carreira \& Barth (2003), Magalhães-e-Silva (2007), Cao et al. (2014) and Radaeski et al. (2014).

We found in this study, for Balfourodendron riedelianum, small to medium pollen grains, with circular amb, subprolate, 3colporate with microreticulate exine. The pollen data are similar to those of Magalhães-e-Silva (2007) for B. molle. In other species of this genus, however, this author classified the exine as striate, as did Barth (1982), which may indicate an ornamentation variation of Baulfourodendron.

Corrêa et al. (1992) described Esenbeckia febrifuga as having medium, prolate, 3-colporate and psilate pollen grains. The results in our study for this species corroborate the previous data; however, we observed subprolate shape and striatemicroreticulate ornamentation.

Although Esenbeckia and Metrodorea are phylogenetically very close (Groppo et al. 2008), the revision of Barth (1982) indicates that these have a distinct pollen morphology, with pollen grains 3-colporate and striate in Metrodorea. We found similar data in Metrodorea nigra in this study, with variation only in the ornamentation, described here as microreticulate.

The Galipea jasminiflora pollen grains were described by Erdtman (1952) as subprolate, 3-colporate and reticulate. Barth (1982) confirmed the results of Erdtman (1952) and also defined the ornamentation of this species as reticulate with numerous bacula inside each lumen. The pollen data introduced by Erdtman (1952) differ from ours in shape (here prolate spheroidal) and aperture (inaperturate). Through the analysis of SEM, we observed bacula inside the lumina of the reticulum as reported by Barth (1982).

The differences in the results previously reported on Galipea jasminiflora and the morphological variations found between the pollen grains of several Galipea species described by Barth (1982), such as G. pentagina A.St.-Hil. with 4-zonocolporate pollen grains and G. bracteata (Nees \& Mart.) Schult. with brevicolporate pollen grains, indicate a eurypalynous genus.

Helietta was cited in the review of Barth (1982) as stenopalynous with prolate, 3-colporate and reticulate pollen grains, with discrete variation in the ornamentation, just as for Zanthoxylum, Pilocarpus and Esembeckia. Radaeski et al. (2014) described Helietta apiculata pollen as subtriangular, subprolate, 3-colporate, lalongate and with a striate-microreticulate exine. Our results corroborate Radaeski et al. (2014), and we observed an ornamentation variation of the apocolpium (striate-microreticulate) and mesocolpium (microreticulate) for these pollen grains, as mentioned by Barth (1982).

Pollen grains of Pilocarpus species were described in Barth's studies (1982, 1985); in general, the author pointed out prolate, 3-colporate and reticulate pollen, similar to those described by Carreira \& Barth (2003) for P. microphylus Stapf. ex Wardlew. Pilocarpus spicatus here analyzed has prolate and 3-colporate pollen grains as described above; however, we observe a striate-reticulate ornamentation, with heterobrochate reticula very evident in the mesocolpium. These details of the pollen surface can be used in species distinctions within Pilocarpus, as noted by Barth (1982) for other genera of Rutaceae.

Barth (1980) classified Zanthoxylum rhoifolium pollen grains as medium, prolate, 3-colporate with exine partially striate, while Corrêa et al. (1992) described them as small, subprolate, 3-colporate and striate-reticulate. Our results are similar to those of Corrêa et al. (1992) but differing in shape, here oblate spheroidal and aperture numbers (4-colporate).

For Zanthoxylum riedelianum and Z. caribaeum (still under the name $Z$. rugosum), Barth (1980) found prolate, 3-colporate and reticulate pollen grains, differing from our results in terms of aperture (4-colporate to $Z$. riedelianum) and ornamentation (striate-reticulate or striate-microreticulate). The pollen grains of Zanthoxylum species have variations in the margo of the apertures, and differences in ornamentation between apocolpium and mesocolpium.

Roubick \& Moreno (1991) classified the pollen grains of Zanthoxylum as circular, subprolate to oblate spheroidal, 3-colporate, reticulate or striate-reticulate. Similar results were reported by Cao et al. (2014), with small to medium pollen grains, spheroidal to prolate, 3-colporate, macroreticulate, striate-rugulate or striate. In our study we observed in the Zanthoxylum species some differences in shape, aperture number and pollen grain ornamentation. We did not identify reticulate pollen grains (as previously mentioned by Roubick \& Moreno 1991), nor macroreticulate or striate-rugulate (as cited in Cao et al. 2014); likewise, previous studies did not observe 4-colporate apertures in pollen grains for the genus, so these palynological data are important to the species identification.

The pollen morphological variation found in the analyzed species and genera of Rutaceae reinforce the importance of palynological studies in Rutaceae. We verified that the quantitative data in some cases could help distinguish the species, as in Galipea jasminiflora (with large pollen grains) and Metrodorea nigra (among colporate species that also have larger pollen grains). For Rutaceae, as well as in other families also analyzed in forest fragments (Souza \& Gasparino 2014; Belonsi \& Gasparino 2015), qualitative data are the main tools in pollen species distinction. 


\section{Conclusions}

(1) The aperture number of Rutaceae pollen grains allows the distinction of inaperturate (Galipea jasminiflora, with apolar pollen grains), 4-colporate (Zanthoxylum rhoifolium and $Z$. riedelianum) and 3-colporate species (the remaining species).

(2) We find different types of pollen ornamentation: microreticulate (Balfourodendron riedelianum), reticulate (Galipea jasminiflora), striate-microreticulate (Esenbeckia febrifuga, Helietta apiculata, Metrodorea nigra and Zanthoxylum riedelianum) and striate-reticulate (Pilocarpus spicatus and all other species of Zanthoxylum).

(3) The species of Esenbeckia, Helietta, Pilocarpus and Zanthoxylum show differences in ornamentation between the apocolpium and mesocolpium of the pollen grains, which helps in the identification of the taxa.

(4) The results obtained from this study demonstrate the pollen diversity of Rutaceae. It is regarded as a eurypalynous family. As mentioned, details of the presence or absence, number of apertures, and ornamentation (and its variations) have contributed to species recognition in the Rutaceae examined.

\section{Acknowledgements}

The authors thank the Universidade Estadual Paulista Unesp (PROPe, RENOVE-2014/211) for the scholarship of the first author and financial support We acknowledge Dr. D.M. Jarzen for critical review of this manuscript and helpful suggestions.

\section{Disclosure statement}

No potential conflict of interest was reported by the authors.

\section{Funding}

This work was supported by the Unesp - Pró-reitoria de Pesquisa - PROPe [grant number RENOVE - 2014/211].

\section{Notes on contributors}

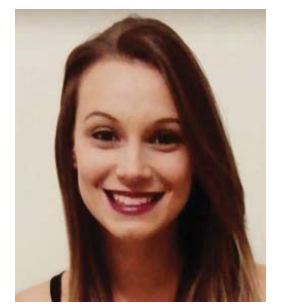

FERNANDA VITORETE DUTRA graduated with a BSC in biological sciences from Universidade Estadual Paulista, UNESP, Faculdade de Ciências Agrárias e Veterinárias, Jaboticabal, Brazil. She is studying for an MSc in comparative biology at the Universidade de São Paulo (USP), Faculdade de Filosofia, Ciências e Letras de Ribeirão Preto, Brazil. Fernanda specialises on the palynology of the Rutaceae, Rubiaceae and Gesneriaceae.

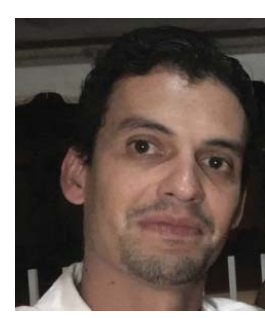

EDUARDO CUSTÓDIO GASPARINO is a professor in the Departamento of Biologia aplicada á Agropecuária of the Universidade Estadual Paulista, UNESP, Faculdade de Ciências Agrárias e Veterinárias, Jaboticabal, Brazil. He received a degree in biological sciences from the Universidade Estadual Paulista, UNESP in 2001. Eduardo also has an MSc and $\mathrm{a} \mathrm{PhD}$ in plant biodiversity and environment from the Instituto de Botânica, Brazil between
2003 and 2008. His research is on the palynology of the Brazilian ecosystems, especially Cerrado and Atlantic Forest.

\section{ORCID}

Eduardo Custódio Gasparino (iD) http://orcid.org/0000-0001-6078-7341

\section{References}

Al-Anbari AK, Barusrux S, Pornpongrungrueng P, Theerakulpisut P. 2015. Pollen grain morphology of Citrus (Rutaceae) in Iraq. International Conference on Plant, Marine and Environmental Sciences (PMES-2015), Jan. 1-2:1-11; Kuala Lumpur (Malaysia).

Appelhans MS, Smets E, Razafimandimbison SG, Haevermans T, Van Marle EJ, Couloux A, Rabarison H, Randrianarivelojosia M, Kebler PJA. 2011. Phylogeny, evolutionary trends and classification of the SpatheliaPtaeroxylon clade: Morphological and molecular insights. Annals Botany 107:1259-1277.

APG IV. 2016. An update of the Angiosperm Phylogeny Group classification for the orders and families of flowering plants: APG IV. Botanical Journal of the Linnean Society 181:1-20.

Arreguín-Sánchez M, Palacios-Chávez R, Quiroz-García DL, Ramos-Zamora D. 1986. Morfología de los granos de polen del género Casimiroa (Rutaceae) del Valle de México. Phytologia 60:391-394.

Barth OM. 1980. Pollen Morphology of a Brazilian Rutaceae. Zanthoxylum (Fagara). Pollen et Spores 22:425-436.

Barth OM. 1982. Variações Polínicas em Espécies Brasileiras da Família Rutaceae. Boletim do Instituto de Geociências 13:129-134.

Barth OM. 1983. Pollen morphology of Brazilian Rutaceae: Dictyoloma and Hortia. Pollen et Spores 25:409-419.

Barth OM. 1985. Pollen morphology of Brazilian Rutaceae: Pilocarpus. Pollen et Spores 27:145-154.

Barth OM, Melhem TS. 1988. Glossário llustrado de Palinologia. Campinas: Universidade Estadual de Campinas; 74p.

Bayer R, Mabberley DJ, Morton C, Miller CH, Sharma IK, Pfeil BE, Rich S, Hitchcock R, Sykes AS. 2009. A molecular phylogeny of the orange subfamily (Rutaceae: Aurantioideae) using nine cpDNA sequences. American Journal of Botany 96:668-685.

Belonsi TK, Gasparino EC. 2015. Pollen morphology of Malpighiaceae from Brazilian forest fragments. Brazilian Journal of Botany 38:379-393.

Breis FB, Sánchez CP, Gilabert E, Castillo ME. 1993. The Pollen Morphology of Citrus limon "Verna" from the Murcia Region, S.E. Spain. Anales de Biologia Universidad de Murcia 19:63-69.

Cao M, Zhang D, Shah A, Dong L. 2014. Pollen Morphology and its Systematic Significance in Zanthoxylum (Rutaceae) from China. Pakistan Journal of Botany $46: 1325-1330$

Carreira LMM, Barth OM. 2003. Atlas de pólen da vegetação de canga da Serra de Carajás, Pará, Brasil. Belém: Museu Paraense Emílio Goeldi $112 p$.

Chase MW, Morton CM, Kallunki JA. 1999. Phylogenetic relationships of Rutaceae: A cladistic analysis of the subfamilies using evidence from $\mathrm{rbcL}$ and atpB sequence variation. American Journal of Botany 86:11911199.

Corrêa AMS, Watanabe HM, Melhem TS. 1992. Flora Polínica da Reserva do Parque Estadual das Fontes do Ipiranga (São Paulo, Brasil). Hoehnea 19:117-124.

Erdtman G. 1952. Pollen Morphology and Plant Taxonomy-Angiosperms. Stockholm: Alquimist e Wiksell; 539p.

Erdtman G. 1960. The acetolysis method. A revised description. Sven Bot Tidskr 54:561-564.

Faegri G, Iversen J. 1966. Textbook of modern pollen analysis. 2nd edn. Copenhagen: Scandinavian University Books; 295p.

Fahrig L. 2003. Effects of habitat fragmentation on biodiversity. Annual Review of Ecology, Evolution, and Systematics 34:487-515.

Fukuda T, Naiki A, Nagamasu H. 2008. Karyotypic analysis of Skimmia japonica (Rutaceae) and related species. Journal of Plant Research 120:113121. 
Gadek PA, Fernando ES, Quinn CJ, Hoot SB, Terrazas T, Sheahan MC, Chase MW. 1996. Sapindales: Molecular delimitation and infraordinal groups. American Journal of Botany 83:802-811.

Gasparino EC, Cruz-Barros MAV, Chautems A. 2013. Pollen morphology in Brazilian species of Codonanthe (Mart.) Hanst. and Nematanthus Schrader (Gesneriaceae). Grana 52:285-274.

Grant M, Blackmore S, Morton CM. 2000. Pollen morphology of the subfamily Aurantioideae (Rutaceae). Grana 39:8-20.

Groppo M, Pirani JR, Salatino MLF, Blanco SR, Kallunki JA. 2008. Phylogeny of Rutaceae based on two noncoding regions from cpDNA. American Journal of Botany 95:985-1005.

Groppo M, Cruz-Barros MAV, Corrêa AMS. 2010. Morfologia polínica de espécies de Hortia (Rutaceae). Revista Brasileira de Botânica 33:13-20.

Kubitzki K, Kallunki JA, Duretto M, Wilson PG. 2011. Rutaceae. In: Kubitzki K, editor. The families and genera of vascular plants, vol. 10 EudicotsSapindales, Cucurbitales, Myrtaceae. Heildelberg: Springer; p. 276-356.

Magalhães-e-Silva FH. 2007. Contribuições à Palinologia das Caatingas. PhD Thesis. Feira de Santana: State University of Feira de Santana; 182p.

McCune B, Mefford MJ. 2011. PC-ORD. Multivariate analysis of ecological data. Version 6. Oregon (USA): MjM Software, Gleneden Beach; 28p.

Melhem TS, Cruz-Barros MAV, Corrêa AMS, Makino-Watanabe H, SilvestreCapelato MSF, Gonçalves-Esteves VL. 2003. Variabilidade polínica em plantas de Campos do Jordão (São Paulo, Brasil). Boletim do Instituto de Botânica 16:1-104.

Morton CM. 2009. Phylogenetic relationships of the Aurantioideae based on nuclear ribosomal DNA ITS region, and three noncoding chloroplast DNA regions, atpB-rbcL spacer, rps16, and trnL-trnF. Organisms Diversity \& Evolution 9:52-68.

Morton CM, Kallunki JA. 1993. Pollen morphology of the subtribe Cuspariinae (Rutaceae). Brittonia 45:286-314.

Morton CM, Grant M, Blackmore S. 2003. Phylogenetic relationships of the Aurantioideae inferred from chloroplast DNA sequence data. American Journal of Botany 90:1463-1469.

Morton CM, Telmer C. 2014. New Subfamily Classification for the Rutaceae. Annals of the Missouri Botanical Garden 99:620-641.

Mou FJ, Zhang DX. 2009. Pollen morphology supports the reinstatement of Bergera (Rutaceae). Nordic Journal of Botany 27:298-304.

Myers N, Mittermeier RA, Fonseca GAB, Kent J. 2000. Biodiversity hotspots for conservation priorities. Nature 403:853-858.

Mziray W. 1992. Taxonomic studies in Toddalieae Hook.f. (Rutaceae) in Africa. Acta Universitatis Upsaliensis Symbolae Botanicae Upsalienses 30:26-33.

Pirani JR, Groppo M. 2016. Rutaceae. In: Forzza RC (coord) Lista de Espécies da Flora do Brasil. Jardim Botânico do Rio de Janeiro. Avaliable from: $<$ http://floradobrasil.jbrj.gov.br/jabot/floradobrasil/FB212>. Acessed Mai 2016.

Poon WS, Shaw PC, Simmons MP, But PPH. 2007. Congruence of molecular, morphological, and biochemical profiles in Rutaceae: A cladistic analysis of the subfamilies Rutoideae and Toddalioideae. Systematic Botany 32:837-846

Punt W, Hoen PP, Blackmore S, Nilsson S, Le Thomas A. 2007. Glossary of pollen and spore terminology. Review of Palaeobotany and Palynology 143:1-81.

Qaiser M, Perveen A. 2005. Pollen Flora of Pakistan (Rutaceae). Pakistan Journal of Botany 37:495-501.

Radaeski JN, Evaldt ACP, Bauermann SG, Lima GL. 2014. Diversidade de grãos de pólen e esporos dos Campos do sul do Brasil: descrições morfológicas e implicações paleoecológicas. Iheringia 69:107-132.

Ranga NT, Rezende AA, Cavasan O, Toniato MTZ, Cielo-Filho R, Stranghetti V. 2012. Caracterização florística de remanescentes de vegetação nativa da região noroeste do Estado de São Paulo. In: Necchi O Jr, editor. Fauna e Flora de fragmentos florestais remanescentes da região Noroeste do Estado de São Paulo. Ribeirão Preto: Holos Editora; p. 105-135.

Razafimandimbison SG, Apelhans MS, Rabarison H, Haevermans T, Rakotondrafara A, Rakotonandrasana SR, Ratsimbason M, Labat JN, Kebler PJA, Smets $E$, et al. 2010. Implications of a molecular phylogenetic study of Malagasy genus Cedrelopsis and its relatives (Ptaeroxylaceae). Molecular Phylogenetics and Evolution 57:258-265.

Ribeiro JF, Walter BMT. 2008. Fitofisionomias do bioma Cerrado. In: Sano SM Almeida SP, Ribeiro J, editors. Cerrado: ecologia e flora. Planaltina: EMBRAPA-CPAC; p. 151-212.
Roubik DW, Moreno JE. 1991. Pollen and spores of Barro Colorado Island. New York: Missouri Botanical Garden; 268p.

Salgado-Labouriau ML, Vanzolini PE, Melhem TS. 1965. Variation of polar axés and equatorial diameters in pollen grains of two species of Cassia. Grana Palynology 6:98-105.

Sato Y. 1971. Pollen morphology of Skimmia Japonica Thunb. Scientific Reports of the Tohoku Univesity, series 4 Biology 35:207-211.

Scott KD, Mcintyre CL, Playford J. 2000. Molecular analyses suggest a need for a significant rearrangement of Rutaceae subfamilies and a minor reassessment of species relationships within Flindersia. Plant Systematics and Evolution 223:15-27.

Shepherd GJ. 1996. Fitopac 1: Manual do Usuário. Campinas: Departamento de Botânica, Universidade Estadual de Campinas; 96p.

Silva Cl, Fonseca VLI, Groppo M, Bauermann SG, Saraiva AM, Queiroz EP, Evaldt ACP, Aleixo KP, Castro JP, Castro MMN, et al. 2014. Catálogo Polínico das plantas usadas por abelhas no Campus da USP de Ribeirão Preto. Ribeirão Preto: Holos; 153p.

Souza CN, Gasparino EC. 2014. Pollen morphology of Fridericia Mart. (Bignoniaceae) from Brazilian forest fragments. Brazilian Journal of Botany 37:83-94.

Specieslink. 2017. Eletronic Database. Available from: http://splink.cria.org. br/. Captured on 10 January 2017.

Thiers B. 2017. Index Herbariorum: a global directory of public herbaria and associated staff. New York Botanical Garden's Virtual Herbarium; [cited 2017 Jan 8]. Available from: http://sweetgum.nybg.org/ih/

Victor JE, Van Wyk AE. 1998. Palynology of Acmadenia (Rutaceae) and its taxonomic implications. Grana 37:143-154.

Victor JE, Van Wyk AE. 1999a. Pollen morphology of Adenandra (Rutaceae) and its taxonomic implications. Grana 38:1-11.

Victor JE, Van Wyk AE. 1999b. Pollen morphology of Coleonema and Diosma (Rutaceae) and its taxonomic implications. Grana 38:12-19.

Victor JE, Van Wyk AE. 2000. Pollen morphology of Phyllosma and Sheilanthera (Rutaceae) and its taxonomic implications. Grana 39:103-107.

Victor JE, Van Wyk AE. 2001. Pollen morphology of Euchaetis and Macrostylis (Diosminae-Rutaceae) and its taxonomic implications. Grana 40:105110.

Vieira S. 2011. Introdução à Bioestatística. Rio de Janeiro: Elsevier; 345 p. Zar JH. 1999. Biostatistical analysis. 4th ed. New Jersey: Prentice Hall; 663 p.

\section{Appendix 1. Specimens examined}

Balfourodendron riedelianum (Engl.) Engl.: Brasil. Paraná: Tabagi, 27 Oct 1995, F. Chagas e Silva \& L.H. Soares-Silva 1859 (SJRP); Brasil. São Paulo: Jaboticabal, 19 Dec 1994, E.A. Rodrigues 254 (JABU).

Esenbeckia febrifuga (A. St.-Hill.) A. Juss ex Mart: Brasil. Paraná: Londrina, 02 Apr 1990, M. F. Gouvea s/n (SJRP); Brasil. Paraná: Rolândia, 25 Nov 1997, V.F. Kinupp 188 (SJRP).

Galipea jasminiflora (A. St.-Hill.) Engl.: Brasil. São Paulo: Matão, 24 Mar 2008, N.T. Ranga 88 (SJRP); Brasil. São Paulo: Matão, 15 Feb 2008, N.T. Ranga s/n (SJRP).

Helietta apiculata Benth.: Brasil. São Paulo: General Salgado, 14 Nov 1999, E. Montilha et al. 59 (SJRP); Brasil. Paraná: Sapopema, 06 Dec 1997, C. Medri \& E. M. Francisco 544 (SJRP).

Metrodorea nigra A. St.-Hill.: Brasil. Paraná: Lobato, 25 Oct 1986, G. Hatschbach 50660 (SJRP).

Pilocarpus spicatus A. St.-Hill.: Brasil. Minas Gerais: Monte Azul, 18 Apr 1996, G. Hatschbach et al. 64970 (SJRP); Brasil. Bahia: Caetité, 21 Apr 1996, G. Hatschbach et al. 65173 (SJRP).

Zanthoxylum caribaeum Lam.: Brasil. São Paulo: Sertanópolis, 29 Sep 1995, A.T. Dias 41 (SJRP). 
Zanthoxylum monogynum A. St.-Hill.: Brasil. São Paulo: São José do Rio Preto, 22 Feb 2006, C.I.G. Moraes s/n (SJRP).

Zanthoxylum petiolari A. St.-Hill. \& Tul.: Brasil. São Paulo: São José do Rio Preto, 23 Nov 1995, V. Stranghetti 542 (SJRP); Brasil. São Paulo: São José do Rio Preto, 20 Nov 2002, S.B. Silva et al. s/ n (SJRP),

Zanthoxylum rhoifolium Lam.: Brasil. Paraná: Teixeira Soares, 02 Oct 1995, A.T. Dias et al. s/n (SJRP); Brasil. São Paulo: Jaboticabal, 02 Oct 1990, E.A. Rodrigues 92 (JABU).
Zanthoxylum riedelianum Engl.: Brasil. São Paulo: Jaboticabal, 19 Dec 1994, E.A. Rodrigues s/n (JABU).

Zanthoxylum rigidum Humb. \& Bonpl. ex Willd.: Brasil. São Paulo: Macaubal, 05 Apr 2008, N.T. Ranga s/n (SJRP); Brasil. São Paulo: Magda, 21 May 2008, N.T. Ranga s/n (SJRP). 